\title{
In Situ Observation of Phase Separation in High-Temperature Superconductor La2- ${ }_{x} \mathrm{Sr}_{x} \mathrm{CuO}_{4}$
}

Jong Seok Jeong ${ }^{1}$, Wangzhou $\mathrm{Wu}^{1}$, Guichuan $\mathrm{Yu}^{2}$, Martin Greven ${ }^{2}$, and K. Andre Mkhoyan ${ }^{1}$

1. Department of Chemical Engineering and Materials Science, University of Minnesota, Minneapolis, Minnesota 55455, United States .

2. School of Physics and Astronomy, University of Minnesota, Minneapolis, Minnesota 55455, United States.

Since the remarkable discovery of superconductivity in 1911 [1], the research of achieving hightemperature superconductivity has been of interest for over a century. Cuprate oxide superconductor only occurred since 1980's because initial studies indicated low-temperature superconductivity, compared to intermetallic compounds [2]. However, these oxide superconductors have quickly become the most heavily studied material system because of its records of high-temperature superconductivity $[2,3]$. One challenge in high-temperature superconductivity is to realize the homogeneity and stability of a material $[4,5]$.

In this work, in situ heating experiments of $\mathrm{La}_{2-x} \mathrm{Sr}_{x} \mathrm{CuO}_{4}(x=0.03)$, as a representative high-temperature cuprate oxide superconductor, is demonstrated in a transmission electronic microscope. So far, there have been many reports about a well-known phase transition in $\mathrm{La}_{2-x} \mathrm{Sr}_{x} \mathrm{CuO}_{4}$ system, which is the transition of the crystal structure from tetragonal to orthorhombic by $\mathrm{CuO}_{6}$ octahedra tilts [6]. Here, on top of the phase transition, we demonstrate a unique phase separation phenomenon based on the analyses of in situ transmission electron microscopy (TEM) and electron energy-loss spectroscopy (EELS).

To obtain high-resolution scanning TEM (STEM) images, an aberration-corrected FEI Titan G2 60-300 STEM, operated at $300 \mathrm{keV}$, was used. Convergence semi-angle of the STEM incident beam was 24.3 mrad and high-angle annular dark-field (HAADF) images were recorded with detector angles of 41-200 mrad. Heated-stage TEM experiments was performed using a Gatan 652 double-tilt heating holder in an FEI Tecnai G2 F30 STEM with TWIN pole piece operating at $300 \mathrm{keV}$ and equipped with a Gatan $4 \mathrm{k} \times 4 \mathrm{k}$ Ultrascan CCD. Bright-field TEM (BF-TEM) images and selected-area electron diffraction (SAED) patterns were acquired using the microscope at each temperature setting. EELS spectra were recorded using a Gatan Image Filter (GIF) spectrometer attached to the microscope.

A phase separation of $\mathrm{La}_{2-x} \mathrm{Sr}_{x} \mathrm{CuO}_{4}$ was monitored by in situ heating experiments in TEM (vacuum level of $\sim 10^{-7}$ Torr). The phase separation took place from as low as $150{ }^{\circ} \mathrm{C}$; however, it occurred mostly in the temperature range of $350-450{ }^{\circ} \mathrm{C}$ (Figure 1). Two resultant phases were identified as metal $\mathrm{Cu}$ and distorted bixbyite $\mathrm{La}_{2} \mathrm{O}_{3}$ by SEAD pattern and EELS analyses. A similar phase decomposition was also reported in $\mathrm{Nd}_{2-x} \mathrm{Ce}_{x} \mathrm{CuO}_{4 \pm \delta}$ system [7]. The existence of the resulting phases after cooling indicates that the phase separation process is irreversible in our experiment setup. The EELS results demonstrated that bulk plasmon peak, $\mathrm{O} K$ and $\mathrm{La} M_{4,5}$ edges change in the temperature range of $350-450{ }^{\circ} \mathrm{C}$ (Figure 2). The bulk plasmon peak from the distorted bixbyite $\mathrm{La}_{2} \mathrm{O}_{3}$ located at $25.2 \mathrm{eV}$ and showed unique $\mathrm{O} K$ edge. We discuss the evolution of crystal structures and EELS peaks as function of temperature in detail [8]. 
References:

[1] KL Chopra and I Kaur, "Thin Film Device Applications" 1983 (Plenum Press, New York).

[2] JG Bednorz and KA Müller, Zeitschrift für Physik B Condensed Matter 64 (1986), p. 189.

[3] G Krabbes et al, "Fundamentals", in High Temperature Superconductor Bulk Materials, 2006 (Wiley-VCH Verlag GmbH \& Co. KGaA, Weinheim).

[4] JW Alldredge et al, Phys. Rev. B 87 (2013), p. 104520.

[5] JC Phillips et al, Rep. Prog. Phys. 66 (2003), p. 2111.

[6] CM Brown et al, Phys. Rev. B 59 (1999), p. 4439.

[7] PK Mang et al, Phys. Rev. B 70 (2004), p. 094507.

[8] This work was supported in part by the NSF MRSEC under award number DMR-1420013, also in part by Grant-in-Aid program of the University of Minnesota.

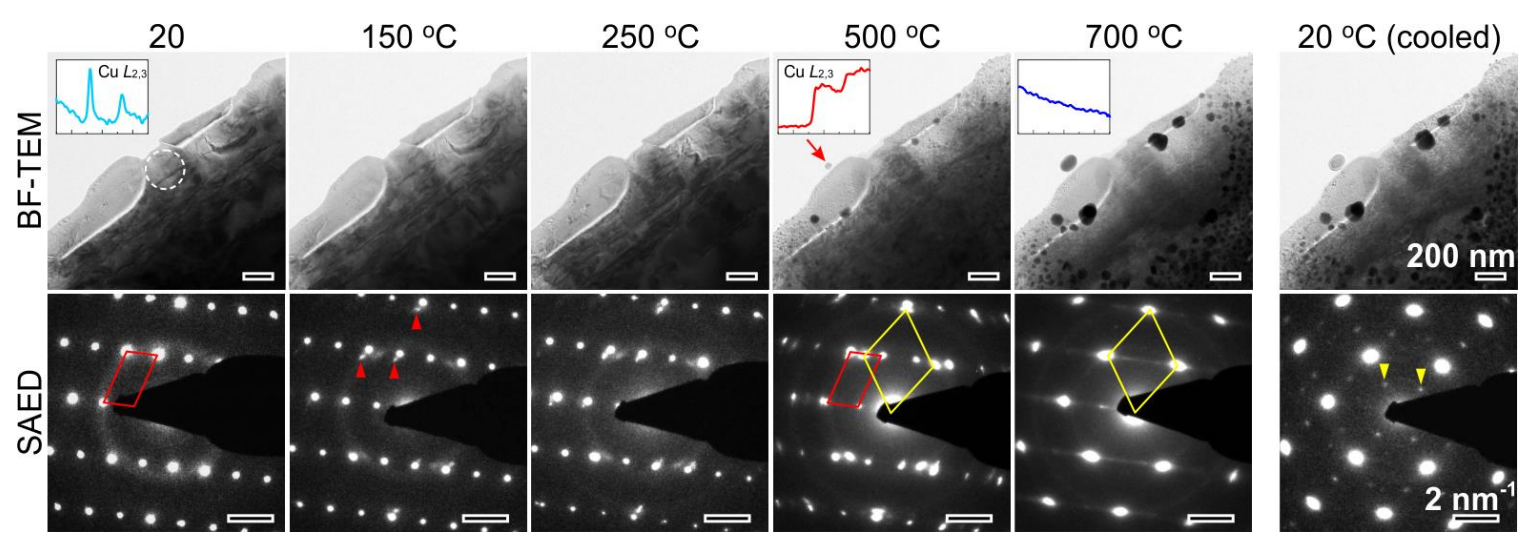

Figure 1. BF-TEM images (top panels) and corresponding SAED patterns (bottom panels) at selected temperatures during a heating experiment. Insets in panels at RT, $500{ }^{\circ} \mathrm{C}$, and $700{ }^{\circ} \mathrm{C}$ are core-loss EELS spectra in the range from 910 to $970 \mathrm{eV}$ of energy loss.
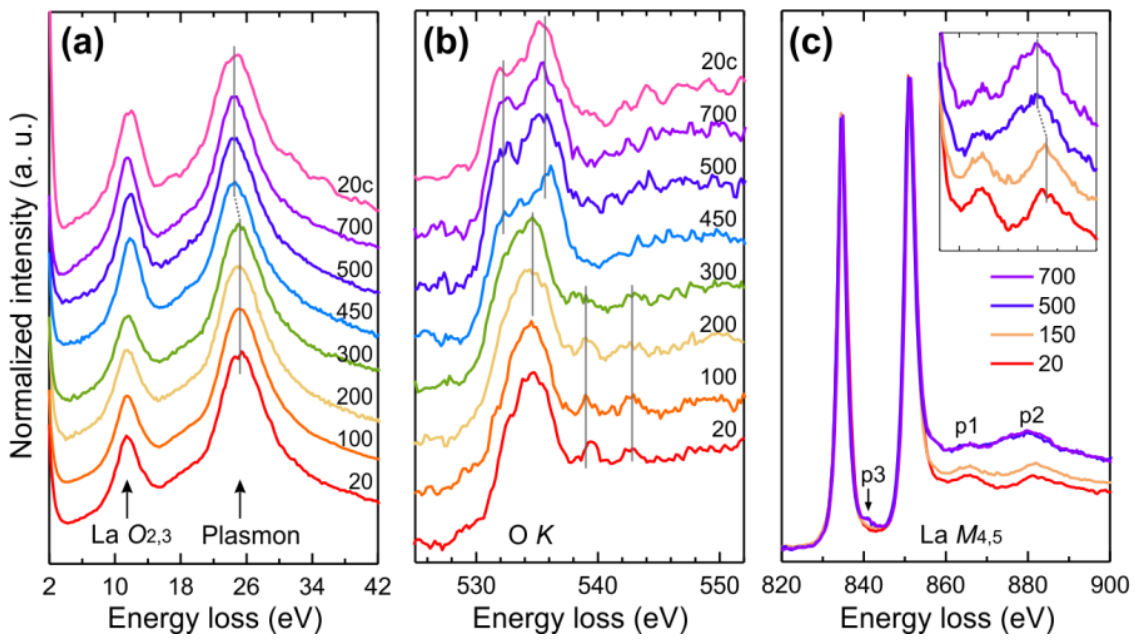

Figure 2. (a) Low-loss, (b) $\mathrm{O} K$ edge, and (c) La $M_{4,5}$ edge EELS spectra at selected temperatures (in ${ }^{\circ} \mathrm{C}$ ) during a heating experiment. Insets in (c) shows magnified EELS spectra in the range of 855-895 eV of energy loss. The $20 \mathrm{c}$ represent the $20^{\circ} \mathrm{C}$ after cooling. 